\title{
Intelligentes Leben in der Stadt der Zukunft
}

\author{
Aliaksei Andrushevich · Kerstin Wessig · Martin Biallas · Rolf Kistler · \\ Alexander Klapproth
}

Eingegangen: 1. März 2015 / Angenommen: 29. April 2015 / Online publiziert: 28. Mai 2015

(C) Springer Fachmedien Wiesbaden 2015

Zusammenfassung Schweizerische Städte rechnen mit Bevölkerungszuwachs zwischen 10-25\% bis zum Jahr 2030. Aufgrund des Platzmangels sind in der Schweiz nur ökologie-bewusste Verdichtungen möglich. Der demografische Wandel ist ein anderer spürbarer Trend. Babyboomers kommen in das Pensionsalter und die Lebenserwartung steigt kontinuierlich weiter bei gleichbleibender Zahl des Pflegepersonals und der Pflegeeinrichtungen. Somit wird die Bevölkerung länger in den eigenen vier Wänden leben, wobei wachsende Ansprüche an Individualität und Selbstbestimmung auch im hohen Alter hinzukommen. Um diesen Herausforderungen gerecht $\mathrm{zu}$ werden und gleichzeitig umweltschonend mit natürlichen Ressourcen umzugehen, sind neue Lösungen für intelligentes Wohnen gefragt. Die Miniaturisierung und das Downscaling als Basis unserer technologischen Entwicklungen heute ermöglicht die nahtlose Integration von Sensoren, Aktoren, Steuerungskomponenten und mikroelektronischen Systemen in smarte Subsysteme, Komponenten und Geräte. Zugleich ermöglichen neue Ansätze des Web 3.0 die Verknüpfung und die Zusammenarbeit verteilter Systeme und Unterkomponenten, die aktiv oder sogar proaktiv Umgebungen gestalten können. Ermöglicht durch die-

\footnotetext{
A. Andrushevich $(\bowtie) \cdot K$. Wessig $\cdot$ M. Biallas $\cdot$ R. Kistler $\cdot$ A. Klapproth iHomeLab, Lucerne University of Applied Sciences and Arts,

Technikumstr. 21,

6048 Horw, Schweiz

E-Mail: aliaksei.andrushevich@hslu.ch

K. Wessig

E-Mail: kerstin.wessig@iHomeLab.ch

M. Biallas

E-Mail: martin.biallas@iHomeLab.ch

R. Kistler

E-Mail: rolf.kistler@iHomeLab.ch

A. Klapproth

E-Mail: alexander.klapproth@iHomeLab.ch
} 
se Gebäudeautomatisierung, sind einerseits geschickte und flexible Aufteilung und Nutzung von Räumen und anderseits energie-effiziente Regelungen ohne Komforteinbußen in Häusern der Zukunft realisierbar. Erfassung der Gewohnheiten eines Benutzers, sowie auf dem Internet der Dinge basierten intelligenten Verknüpfung von Daten aus verschiedenen Lebensbereichen außerhalb eines Gebäudes soll Lebensqualität in Zukunft weiter erhöhen. Diese neuen, smarten Systeme sollten als Anforderung an eine Gestaltung einer Stadt der Zukunft dabei jedoch einen mehrfachen Mehrwert erzielen: sie sollten in der Lage sein, sowohl zur Intensivierung der Human-Building-Interaction, zur Erhaltung und Förderung von Mobilität, zur Senkung des Energieverbrauchs als auch zur Senkung der Kosten zur Gesunderhaltung beizutragen.

Schlüsselwörter Assistenzsysteme $\cdot$ Sicherheit $\cdot$ Intelligentes Wohnen $\cdot$ Ambient Assisted Living · Gebäudeintelligenz · Internet der Dinge

Abstract Swiss cities expect population increase between $10-25 \%$ by the year 2030. Due to lack of space only ecology-conscious construction sides are yet possible in Switzerland. Demographic change is another noticeable trend. The baby boomers start to enter the retirement age now and life expectancy continues to increase while a number of nurses and nursing facilities remains nearly constant. Thus, the population will live longer in their own homes, with growing demands for individuality and self-determination even in high age. To meet these challenges while to be an environmentally friendly with natural resources use, new solutions for intelligent living are required. Miniaturization and downscaling as the basis of our technological developments now allows seamless integration of sensors, actuators, control components and microelectronic systems in smart subsystems, components and devices. At the same time, new approaches of Web 3.0 allow to link and orchestrate the distributed systems and sub-components in such a way that environments can become active or even proactive. Optimal and flexible usage of shared spaces as well as energy-efficient control strategies are becoming possible by the implemented building automation without sacrificing comfort in future homes. Gathering the habits of a user, and intelligent combination of data from different life activities outside of a building are designed to enhance the quality of life in the future. The new based on the Internet of Things smart systems should achieve multiple added values: they should be able both to intensify the Human Building Interaction, promote mobility while reducing energy consumption as well as helping to reduce the costs for maintaining good health. These new smart systems must be used as a design requirements for the cities of future.

\section{Einführung}

In 2020 werden $20 \%$ der Weltbevölkerung über 65 Jahre alt sein (Börsch-Supan und Mariuzzo 2005). Gesellschaften mit hohem Altersdurchschnitt führen zu einem reduzierten Anteil von Erwerbstätigen. Bei einem gleichzeitigen kontinuierlichen Anstieg der Lebenserwartung erhöht sich zudem für die Hochalten das Risiko der Pflegebe- 
dürftigkeit (Doblhammer et al. 2005). Insbesondere in ländlichen Regionen der westlichen Welt findet sich bereits heute eine deutliche Ausdünnung der Bevölkerung bei gleichzeitiger Zunahme des relativen Anteils der Älteren. Ein an sich prosperierendes Wirtschaftswachstum - auch im Gesundheitswesen - wird hierdurch erheblich eingedämmt oder geht zurück, da die Zahl der in einer Region kulturell und strukturell integrierten Arbeitskräfte - ebenfalls im Gesundheitswesen - kontinuierlich abnimmt (Wessig 2012).

In Deutschland steigt die Zahl der Kleinsthaushalte immer noch deutlich. Hingegen nimmt die Zahl der Haushaltsmitglieder ab. Lebten 2010 noch durchschnittlich 2,03 Personen in einem Haushalt, so werden 2030 nur noch 1,88 Personen sein (Entwicklung der Privathaushalte 2011). Diese Veränderung bringt einen Anstieg der Ein- bzw. Zweipersonenhaushalte mit sich. Stirbt in einem Zweipersonenhaushalt ein Bewohner, bleibt die andere Person zumeist weiterhin in der Wohnung wohnen. Langjährig bestehende Haushalte werden somit typischerweise beibehalten, auch wenn die Größe für die aktuelle Lebenssituation nicht mehr unbedingt benötigt wird. Eine Veränderung erfolgt erst, wenn finanzielle oder gesundheitliche Restriktionen so stark werden, dass ein Umzug zwingend wird. Die steigende Zahl an Kleinsthaushalten ist aber nicht nur auf die Zunahmen von allein lebenden Personen im Alter, sondern auch auf Individualisierung und die Zunahme an Singles zurückzuführen.

Durch die fortschreitende Individualisierung und den gesellschaftlichen Wandel sind gesellschaftliche und soziale Bindung nicht mehr gegeben bzw. selbstverständlich. Die Bestimmung der eigenen Position in der Gesellschaft wird zum zentralen Thema im Alter. Die soziale Verortung verweist auf den Ort, an dem sich eine Person aufgehoben fühlt. Für die Teilnahme am gesellschaftlichen Leben spielen die Kommunikation und der gegenseitige Austausch eine entscheidende Rolle. Die Wahl der Kommunikationsmittel wie persönliche Treffen, Telefon und Internet unterliegen gleichermaßen den persönlichen Präferenzen. Die Häufigkeit des Austausches mit Familienangehörigen, Freunden und Nachbarn bestimmt die soziale Verortung. Somit kommt der Kommunikation und den technischen Kommunikationsmitteln eine sehr große Bedeutung zu (Sayago et al. 2011). Treten im höheren Alter Einschränkungen in der Mobilität auf, werden die technischen Kommunikationsmittel Grundvoraussetzung für die soziale Verortung.

Anderseits bewies Gampe, J. (Maier et al. 2010), dass sich trotz stetiger Zunahme von erreichten Lebensjahren, körperliche und geistige Gebrechen ins hohe Lebensalter verschieben und sich unsere Gesellschaft trotz Alterung eher verjüngt, da die derzeit jungen Alten viele gesunde Lebensjahre bis zum Eintritt von Pflegebedürftigkeit vorweisen, insbesondere, wenn diese zeitlebens körperlich und vor allem geistig aktiv waren. Groß angelegte Studien in Japan in über Jahrzehnte aufgebauten Silver Human Ressource Centers zeigen, dass soziale Teilhabe, Lebensqualität, geistige und körperliche Gesundheit bis ins hohe Alter durch koordinierte, angeleitete und aktive Einbindung in sinnvolle und für sie zu bewerkstelligende Arbeitsaufgaben positiv beeinflusst werden kann (Naganawa 1997). 


\section{Altersbedingte Besonderheiten der Bevölkerung}

Im Alter ist der Erhalt bzw. die Steigerung der Lebensqualität ein wichtiges Thema. Wurden in jungen Jahren noch Einschränkungen bei der häuslichen Lebensqualität in Kauf genommen, werden Faktoren wie Komfort und Sicherheit im Alter immer wichtiger. Erst mit zunehmendem Alter werden die Themen wie finanzielle Absicherung, sich etwas leisten zu können, Luxus, Gesundheitsförderung und Bildung immer wichtiger. Grundsätzlich ist die Tendenz zu beobachten, dass im Alter die Früchte der eigenen Arbeit und des persönlichen Engagements geerntet werden möchten. Dies gilt gleichermaßen für die berufliche Tätigkeit, die eigenen Interessen, die Hobbys sowie für die Freizeitgestaltung. Die Steigerung des Komforts beginnt oftmals mit kleinen Veränderungen in der eigenen Wohnung. Dies begründet sich dadurch, dass schon mit relativ geringen finanziellen Mitteln eine deutliche Steigerung möglich ist (z. B. anschaffen eines größeren Fernsehers).

Mit zunehmendem Alter wird die Wohnung immer mehr zum Lebensmittelpunkt (Speziell im hohen). Ältere Personen leben oftmals seit vielen Jahren in der gleichen Wohnung und haben eine starke emotionale Verbindung zu dieser. Das gewohnte Quartier sowie die Nachbarn sind weiter Faktoren, welche die emotionale Bindung erhöhen. Die Wohnung wird zum Symbol für Intimität, individuelle Freiheit, Sicherheit und Erfolg. Die Wohnung verleiht den Bewohnern einen positiven Status. Erinnerungen an viele schöne Stunden, emotionale Momente und die persönliche Gestaltung geben Sicherheit und ein Gefühl des Wohlbefindens.

\section{Mehrwert durch technische Vernetzung}

Allein durch den technischen Fortschritt ist das Potenzial zur Steigerung der Lebensqualität noch nicht ausgeschöpft. Technischer Fortschritt führt erst dann zu einer Steigerung der Lebensqualität, wenn die Technik an sich in den Hintergrund tritt. Die Funktion eines Gerätes muss sich in den alltäglichen Tagesablauf der Bewohner nahtlos einpassen. Häusliche Assistenzsysteme werden die größte Akzeptanz erreichen, wenn diese einfach und hilfreich sind. Simplizität drückt sich dadurch aus, dass nicht die Technik, sondern nur die gewünschte Funktionalität wahrgenommen wird.

Ein großer Mehrwert entsteht in einem Haushalt zum Beispiel, wenn die Geräte untereinander intelligent vernetzt sind. Die Vernetzung von Sensoren und Geräte sowie die Einführung neuartigen Informationsquellen mit den Möglichkeiten ein Smart Home zu realisieren scheinen heute schon beinahe endlos zu sein. In der Theorie lässt sich alles mit allem vernetzen. Die Realität in den Wohnungen sieht jedoch anders aus. Die Infrastruktur in bestehenden Gebäuden ist auf einem veralteten Stand und bis heute weitgehend unsaniert. Selbst bei Neubauten werden in den Wohnungen kaum intelligente Geräte mit integrierten Netzwerkschnittstellen verbaut. Einzig im Bereich der Energieeinsparung bei Heizungen und Beschattung kommen Gebäudeautomatisierungssysteme zum Einsatz. Berücksichtigt man, dass der Renovationszyklus eines Hauses bei ca. 40 Jahren liegt, bedeutet dies, dass Gebäude, welche heute saniert werden, in Zukunft weiterhin keine Infrastrukturvorteile bieten. Assistenzsysteme der zukünftigen Generation können folglich keine leistungsfähige Infrastruk- 
tur im Wohnumfeld erwarten. Als Konsequenz sollten häusliche Assistenzsysteme zukünftig derart gestaltet werden, dass sie nachträglich und für einen bestimmten Zeitraum in eine Wohnumgebung eingebaut werden können. Erschwerend kommt hinzu, dass zu viele technische Standards für eine häusliche Vernetzung existieren (IFA) Verschiedene häusliche altersgerechte Assistenzsysteme, welche Wohnstil, Lifestyle und Unterstützung der Einwohner nahtlos kombinieren, sollen künftig zur Lösung der mit dem demografischen Wandel verbundenen Herausforderungen beitragen.

\section{Vorteile der Gebäudeintelligenz}

Die heutigen Automatisierungssysteme der Gebäudesteuerung bieten vielfältige Möglichkeiten Raumklima, Beleuchtung und die Präsentation von multimedialen Inhalten zu steuern. Die Steuerung der sogenannten Raumparameter erfolgt meist nach festen Regeln, welche einmal programmiert werden. Eine typische Regel ist die Nachtabsenkung der Heizung. Solche Regeln lassen sich zumeist nicht oder nur bedingt von den Bewohnern einer Wohnung an die individuellen Bedürfnisse anpassen. Besonders im Krankheitsfall oder z. B. bei Schlafproblemen wäre es sinnvoll, dass die Raumparameter wie Heizung an die aktuelle Situation angepasst werden könnten. Einige wenige Systeme bieten inzwischen Funktionen an, welche die Steuerungsregeln an die Lebensgewohnheiten der Bewohner anpasst. Diese Systeme erkennen die Lebensgewohnheiten und steuern Beleuchtung oder Heizung entsprechend. Die vom Bewohner als komfortabel empfunden Raumparameter werden nur dann eingestellt, wenn der Raum auch genutzt wird. Solche Systeme benötigen eine Grundmasse an Daten für die Entscheidungen. Solche Daten können der aktuelle Strombedarf, Wasserverbrauch oder Daten von anderen Raumsensoren sein. Da oftmals diese Informationen in einer Wohnung nicht zur Verfügung stehen, benötigt es andere Datenquellen. Heutige Funktechnologien ermöglichen es, dass Signale von bestehenden Schaltern nutzbar gemacht werden und einem intelligenten System zur Verfügung gestellt werden. Diese Funkmodule lassen sich problemlos in jede Art von Schalter nachträglich einbauen. Mit den Signalen von den Schaltern wird der Lichtbedarf einer Person ermittelt. Nach einer gewissen Lernphase kann das Steuerungssystem automatisch die Beleuchtung entsprechend der Lebensgewohnheiten einstellen. Dies ist nur ein mögliches Beispiel, wie Gebäudeintelligenz mit einer intuitiven, einfachen und nahtlosen Bedienbarkeit in bestehenden Gebäuden und Wohnungen nachgerüstet und genutzt werden kann. Werden möglichst viele Raumparameter intelligent gesteuert, steigt der Wohnkomfort an. Dank der Gebäudeintelligenz steigt nicht nur der Komfort in der eigenen Wohnung, es erhöht sich zusätzlich die Lebensqualität.

\section{Mobile Revolution}

Digital Natives sind heute nicht mehr ausschließlich in der jüngeren Generation zu finden, sondern nehmen insbesondere auch in der sehr heterogenen Gruppe der 
Älteren stark zu. Die Einschätzung, Ältere eindimensional aufgrund ihres Alters einfach den digitalen Immigranten zuzurechnen, ist veraltet. Vielmehr wird heute dem Umstand Rechnung getragen, dass Technikaffinität nicht ausschließlich altersabhängig, sondern eher Bildungs - und geschlechtsabhängige Faktoren aufweist.

Durch den gezielten Einsatz mobiler Anwendungen können durch den Alterungsprozess abnehmende kognitive und physische Fähigkeiten teilweise kompensiert werden. Ferner können die auf Berufserfahrung, und die auf besonderen Fertigkeiten der Älteren basierende Leistung, durch die Bereitstellung mobiler internetbasierter Technologien gesteigert werden. (Maier et al. 2010).

Insbesondere in strukturschwachen Räumen können Informationen auf mobilen Geräten, intelligent ausgewertet, die Versorgung (z. B. Lebensmittel) und die Mobilität durch Verbesserung des Zugangs zu Versorgungssystemen erhöhen und den Zugang zum Gesundheitssystem sichern und so die Infrastruktur der Region verbessern (Schaible et al. 2007).

Hierdurch können sozialverträgliche familiäre Strukturen, Humanvermögen und wirtschaftliche Leistungsfähigkeit in den Regionen erhalten werden und ein großes Wertschöpfungspotenzial für Unternehmen, die solche Lösungen als Produkte, Services bzw. komplexe soziotechnische Systeme anbieten, entstehen (Sundín 2009).

Ein möglicher Lösungsansatz bei der Erhaltung der Selbstständigkeit, geistigen und körperlichen Aktivität und Selbstbestimmtheit älter werdender Menschen in ihrem Lebensumfeld wird durch mobile, technische und gleichzeitig sozial integrierte Internet-Applikationen und Systeme gesehen (Mattern 2007). Wobei der Mensch bei diesen Systemen immer im Zentrum steht. Zudem können intelligent vernetzte Sensoren und entsprechende Mobilitätskomponenten und deren Einbindung in innovative Geschäftsmodelle wesentlich zur Förderung und Erhalt von gesundheitsfördernden Lebensumgebungen, sowie zur Förderung der Autonomie und der nahtlosen Mobilität beitragen.

Viele Ältere managen heute nicht selten Blogs oder Wikis und entwickeln selbst Anwendungen des Web 3.0. Daher wächst der Markt für die Nutzung und die Zusammenführung mobiler, strukturierter Daten, Anwendungen und Umgebungsinformationen in der mobilen Anwendung insbesondere im persönlichen, Gesundheits- und im Dienstleistungsbereich rasant (Mattern 2007).

Durch Vernetzung und Integration vorhandenen technischen Wissens, handwerklicher Fähigkeiten, und gewachsener Unternehmensstrukturen können mobile Produkt-Dienstleistungs-Systeme angeboten werden, die es älter werdenden Menschen ermöglichen, ihre Selbständigkeit im gewohnten Umfeld zu erhalten und trotz abnehmender physischer und kognitiver Leistungsfähigkeit die Teilhabe am Familienleben, in der Gesellschaft und eventuell sogar am Arbeitsleben (beispielsweise durch Heimarbeit) zu gewährleisten [Voelcker-Rehage et al. 2010).

Mobile, sensorgestützte Informations- und Kommunikationssysteme unterstützen durch die Bereitstellung - an den Bedarf und die Einschränkungen der jeweiligen Benutzer individuell angepasste - technische Servicefunktionen und helfen gleichzeitig, durch ihren Betrieb Energie einzusparen.

Hierzu gehören mobile Smart Home Anwendungen genauso wie mobile Gesundheitsapplikationen, aber auch mobile Einkaufs - Logistik - und Arbeitssysteme, die 
Heimarbeit ermöglichen und die durch empirische Untersuchungen, Nutzerstudien, Benutzbarkeitstests, Wohnraumstudien evaluiert werden müssen (Erdt et al. 2011).

Insbesondere Technologien und Produkte für das häusliche Wohnumfeld werden bereits heute und zukünftig vermehrt nachgefragt:

- Von Notrufsystemen über Tele Monitoring-Systeme bis hin zu mobil steuerbaren Anlagen für Energie und Haushaltsgeräte, soziotechnische Dienstleistungen und Technologien und Systemlösungen für die Versorgung mit Gütern, nicht nur des täglichen Gebrauchs, sondern

- Technologien zur Sicherung sozialer Kontakte, Kommunikation, Entwicklung sozialer Organisationen, Sicherung sorgender Gemeinschaften, zum vereinfachten Umgang mit Behörden und insgesamt der Verbesserung der Teilhabe am kulturellen Leben.

Die Befriedigung dieser Bedürfnisse und die Deckung dieses Bedarfs ist Voraussetzung dafür, dass Ältere in ihrer gewohnten Umgebung bleiben und damit die Entwicklung ihrer Kommunen stützen. Wenn es gelingt, diesen Bedarf aus der Region heraus zu decken, ergibt sich zugleich ein neuer Beschäftigungseffekt in der Region, der die regionale Entwicklung zusätzlich stützt. Wenn es darüber hinaus gelingt, auch einen Teil der erforderlichen (technischen) Innovationen in der Region zu erzeugen, erhöht sich der Entwicklungsimpuls für die Region (Schaible et al. 2007; Mattern 2007).

\section{Fallbeispiel: Autonomie im Alter}

Autonomie im Alter ist ein erstrebenswerter Zustand unserer älter werdenden Gesellschaft. Vor allem der Bereich medizinische Sicherheit ist häufig ein Grund warum alleinstehende ältere Menschen das eigene Heim verlassen und sich in die Obhut eines Pflegeheims begeben müssen. Mit dem Projekt iHomeRescue wird ein Sicherheitssystem demonstriert, welches Unfälle im häuslichen Umfeld erkennt und darauf intelligent reagiert. Der Vernetzung mit einer Rettungsleitstelle eines Notfalldienstes kommt bei diesem Sicherheitskonzept eine besondere Bedeutung zu. Die dazu am iHomeLab entwickelten und demonstrierten Techniken umfassen einen Sturzsensor, die Indoor-Personenlokalisierung und eine Webcam, die ein Life Video der Situation übermittelt. In der Rettungsleitstelle kommt ein multi-user und multi-touch fähiges Display zum Einsatz.

\section{Sicherheitsrisiko Sturz}

Ein Sturz stellt - und dies nicht nur im Alter - ein erhebliches Unfallrisiko dar. In der Schweiz werden mehr Personen durch Sturzunfälle verletzt als durch Verkehrsunfälle. Dabei passieren die meisten Unfälle im häuslichen Umfeld. Ein mehrstufiges Sicherheitskonzept, genannt iHomeRescue, soll helfen den Sturz zu erkennen und nötigenfalls eine Notfallversorgung durchzuführen Die Hilfeleistung umfasst die Benachrichtigung nahestehender Personen, Unterstützung der Kontaktaufnahme, 
sowie bei Bedarf die Einleitung der Rettungsmaßnahmen durch Kontaktierung einer Notfall-Leitstelle.

\section{Eingesetzte Technologien}

Da die Funktion des iHomeRescue am Beispiel einer gestürzten Person verdeutlicht werden soll, kommen hier die nachfolgend beschriebenen Basiskomponenten zum Einsatz. Zum einen ein zu diesem Zweck entwickelter Sturzsensor, das Lokalisierungssytem eLoc und der iHomeTable, eine interaktive, multitouchfähige Bildschirmschnittstelle.

Um den Sturz zu erkennen, wurde der Sturzsensor iCare entwickelt [Abb. 1], welcher mit der low-power Drahtlostechnologie, basierend auf IEEE802.15.4/ZigBee im ISM Band auf 2,4 GHz kommuniziert, und gegebenenfalls einen Sturzalarm auslöst (Andrushevich et al. 2009). Auf der Anwendungsschicht wird ein proprietäres Protokoll verwendet. Das neue, von ZigBee für solche Anwendungen vorgeschlagene Profil wird jedoch noch von keinem Hersteller offiziell eingesetzt. Durch den niedrigen Strombedarf wird mit einer Lithium-Ionen-Batterie (160 mAh) eine Betriebszeit des Sensors von bis zu 4 Jahren erreicht.

Der Sturz wird dabei aufgrund einer Kombination von Luftdruckveränderung (Höhenmessung) und Beschleunigungsmustern ermittelt und durch einen Mustererkennungsalgorithmus zuverlässig detektiert. Der Luftdrucksensor hat einen Messbereich von 0 bis $2000 \mathrm{hPa}$ und eine relative Messgenauigkeit von ca. $20 \mathrm{~cm}$ bei einer Messdauer von $1 \mathrm{~s}$. Der Beschleunigungssensor arbeitet mit 3 Achsen und bis $8 \mathrm{~g}$.

Zusätzlich ist auf dem Modul ein Temperatursensor integriert, der ohne Kalibrierung auskommt und eine absolute Genauigkeit von 1 Grad aufweist. Bei Bedarf

Abb. 1 Der Sturzsensor iCare

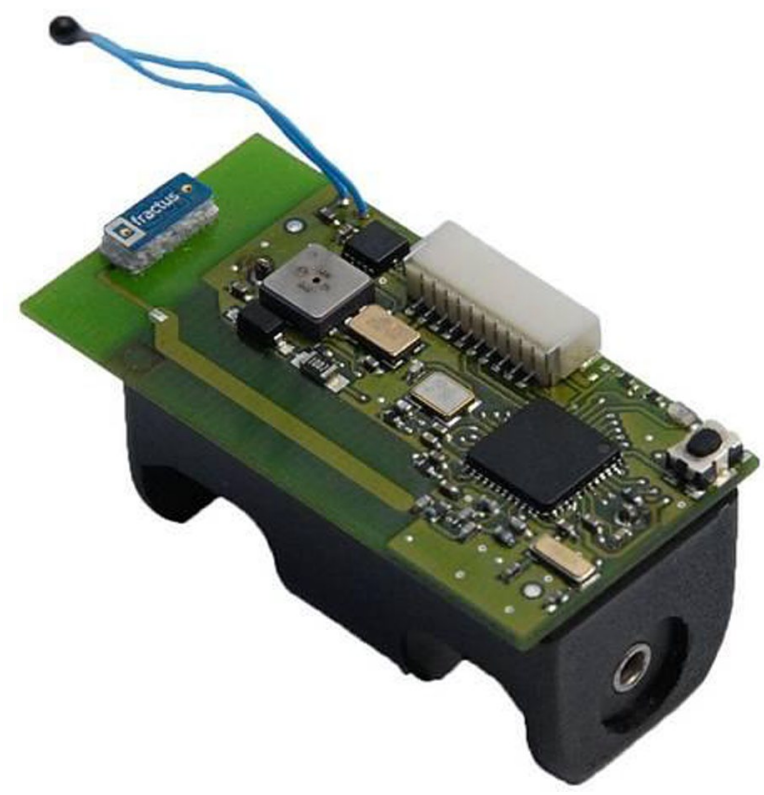


kann das Modul mit weiteren Sensoren, zum Beispiel für Biomonitoring ausgerüstet werden.

Bei einem Einsatz eines öffentlichen notärztlichen Dienstes ist zudem an eine Zutrittsmöglichkeit zu den Räumlichkeiten der verunfallten Person zu denken. Türsysteme werden immer einbruchssicherer und ohne Hausmeisterservice ist eine automatische Türöffnung von Vorteil. Hier kommt der Sprachsteuerung im Bereich der Human-Building-Interaction - von der verunfallten Person zum System bzw. vom Notfalldienst zum System, beispielsweise mit einem zuvor übermittelten Codewort - eine besondere Bedeutung zu. Diese Art der Sprachsteuerung ist bereits heute ein Bestandteil des iHomeLabs und kann auf die entsprechenden Erfordernisse angepasst werden.

Eine weitere Neuerung, die hier Anwendung in einer Notfallzentrale findet, stellt die Art der Nutzung neuer interaktiver Technologien, wie beispielsweise die des Microsoft Surface Tables (Abb. 2) dar. Unsere Evaluationen haben ergeben, dass der Surface vor allem durch die von Microsoft verfügbare Entwicklungsumgebung, den Support und die Fähigkeit mit Geräten auf dem Tisch zu interagieren, die ideale Plattform für das iHomeRescue Projekt bietet. So ist er fähig, per LAN oder Bluetooth

Abb. 2 Einsatz des multitouchfähigen iHomeTable in der Notrufzentrale

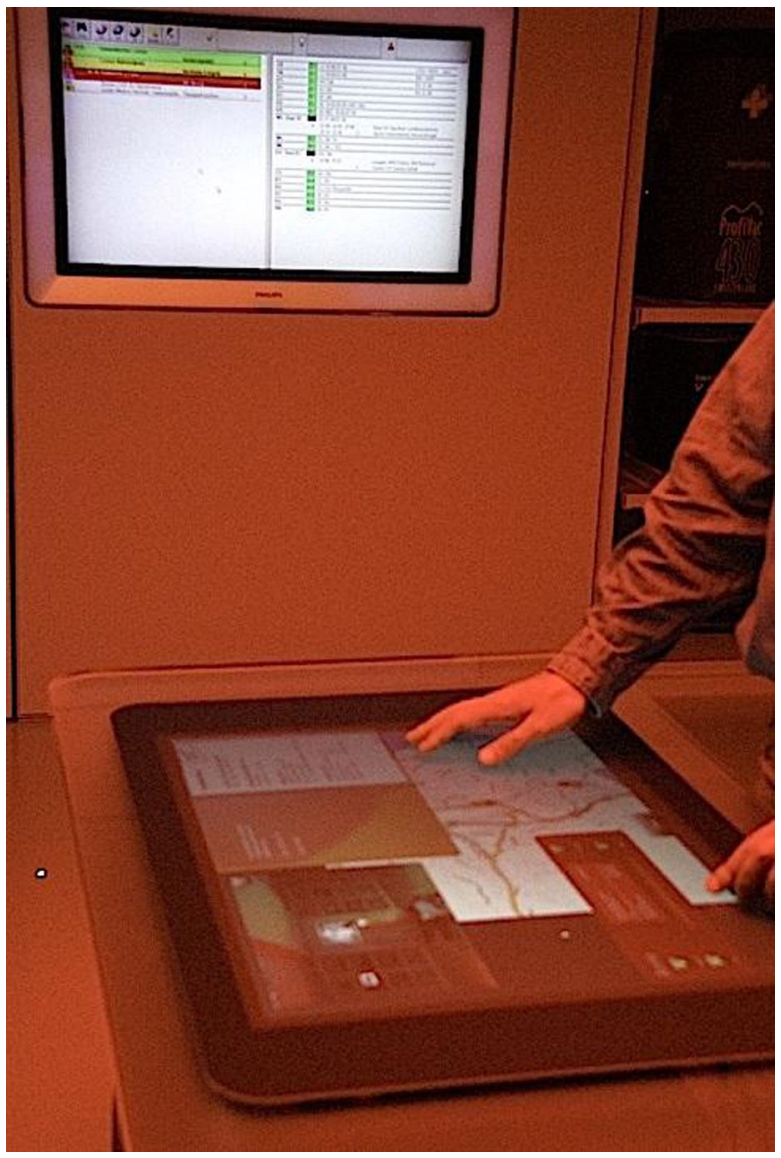


mit der Umwelt zu kommunizieren. Zudem wird eine touchsensitive Bildschirm- und Tischfläche geboten, die von allen Seiten und von mehreren Personen gleichzeitig genutzt werden kann. Die Idee eines Bedientisches (mit und ohne Multitouch-Fähigkeit) existiert bereits seit einiger Zeit, doch erst kürzlich ist solche Hardware kommerziell erhältlich. Diese Erweiterung zur Rundumnutzung (multipersonelle Bedienung) erfordern neue Ansätze bei der Gestaltung und Nutzung von Benutzeroberflächen damit diese ihre Vorteile, wie bei einer Einsatzplanung in einer Notrufzentrale entfalten können. Hier - wie auch bei dem gesamten Projekt - zeigt sich, dass eine einfache, intuitive Benutzerschnittstelle maßgeblich zur Akzeptanz eines technischen Systems oder einer Applikation bei den Anwendern beiträgt.

\section{Sicherheitskonzept zur Unterstützung der Autonomie im Alter}

Ein Softwaresystem welches verschiedene Szenarien kennt und so auch Fehlalarme erkennen kann, hat daraufhin mehrere Möglichkeiten entsprechend zu reagieren. Zunächst muss der Alarm bekannt - also weitergeleitet - werden. Dies geschieht im Allgemeinen über Telefon an eine vorgegebene Person einer Notfallliste. Ist diese nicht erreichbar oder reagiert sie nicht, so wird jeweils versucht die nächste Person der Liste zu benachrichtigen. Zusätzlich kann auch eine SMS zur Benachrichtigung versendet werden. Für eine Kontaktaufnahme mit der verunfallten Person können Lautsprecher und Mikrofone der Umgebung genutzt werden.

Besonders interessant ist sicher auch die Aufnahme von Versorgungsakteuren in die persönliche Notfallliste, wie z. B. die Sanitätsnotrufzentrale, die in der Schweiz unter der Nr. 114 erreichbar ist. Ein Modellversuch ist bereits in Planung. Diese erhalten die Meldung innerhalb ihres Softwaresystems (Abb. 3).

Um Position der Personen im Notfall (z. B. beim Sturz) automatisch zu identifizieren, kommen zwei Lokalisierungssysteme in Gebäuden zum Einsatz. Dabei wird zwischen einem stationären und einem mobilen System unterschieden. Stationär wird Ultraschall eingesetzt und zur mobilen Unterstützung ein Roboter verwendet.

Eine genaue Lokalisierung kann über das am iHomeLab entwickelte und auf Ultraschall basierendes System eLoc geschehen (Knauth et al. 2009). Hierbei handelt es sich um ein System zur automatischen Positionsbestimmung von Geräten der Gebäu-

Abb. 3 Sturzdetektion in der Notrufzentrale

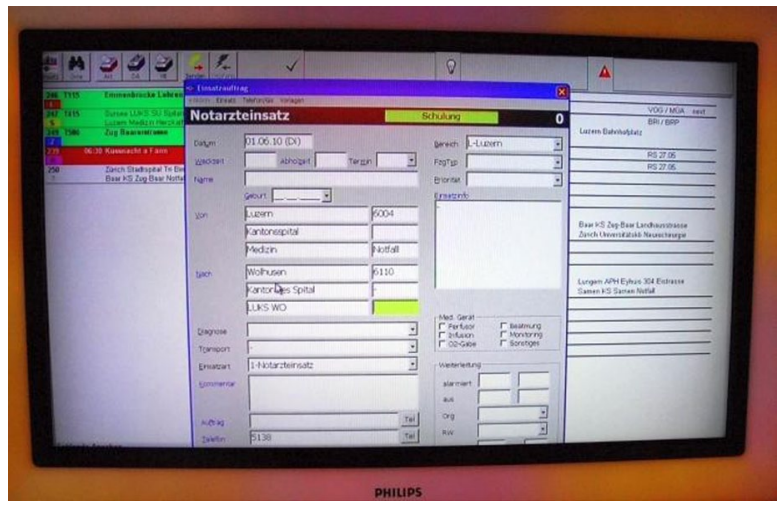


Abb. 4 a Lokalisierung mit Ultraschall: Empfänger. b Lokalisierung mit Ultraschall: Vorder- und -rückseite - hier in ein Batch integriert - mit einem Ricola als Vergleich
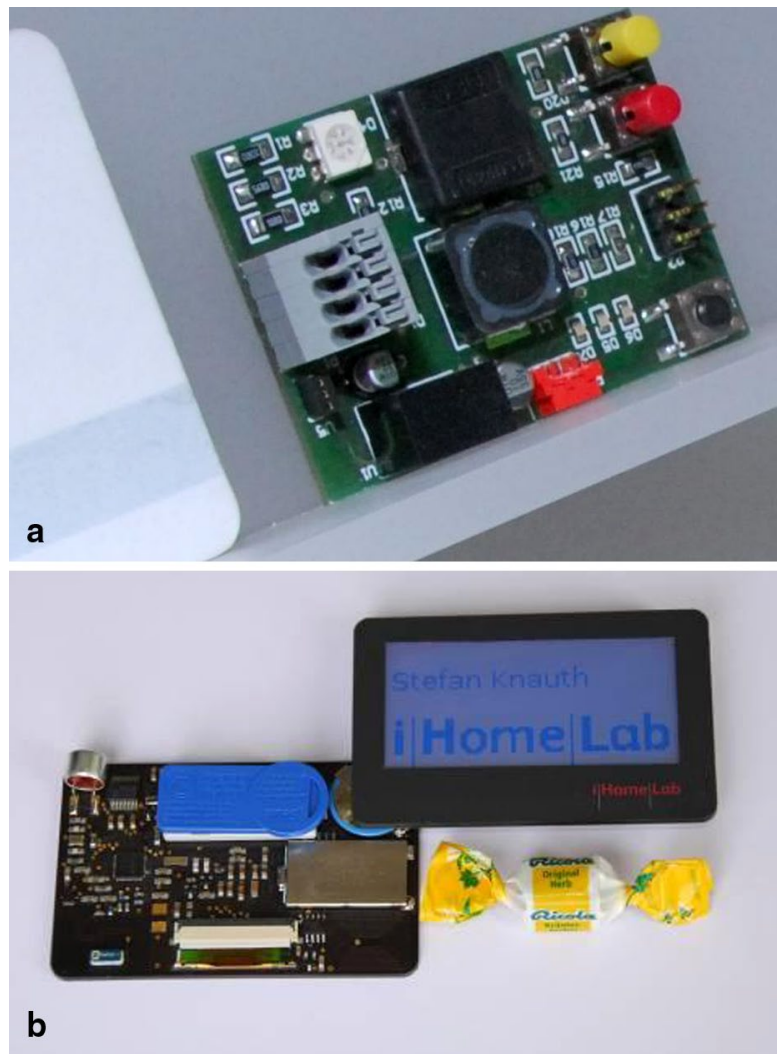

deautomation und somit auch von Personen, die mit einem solchen Sender ausgestattet sind. Die Sender können sich bei der Kenntnis ihrer Position und des räumlichen Kontexts in das System einbinden und aktuelle Positionsdaten senden sowie Aktionen auslösen. Der entstandene Prototyp (siehe Abb. 4a, b) hat gezeigt, dass es mit Hilfe von IEEE802.15.4 Funk und Ultraschallwellen möglich ist, die Sender auf bis zu $10 \mathrm{~cm}$ genau (gegenüber im besten Fall $1 \mathrm{~m}$ Genauigkeit bei heutigen Lösungen, die auf WLAN und Beacons basieren) zu lokalisieren. Dabei können zurzeit bis zu 10 Objekte in einem Gebäude gleichzeitig verfolgt werden.

Zudem wird im Showcase des iHomeLabs der Hochschule Luzern bereits auf einen Roboter gesetzt, der über ein Webinterface zum Unfallort gesteuert werden kann und über seine Web-Kamera Informationen über den Unfallort und die Situation liefern kann. Diese Information kann von den verständigten Vertrauenspersonen, aber auch vom Rettungsdienst verwendet werden. Der Roboter arbeitet zu seiner Orientierung neben der Anbindung an das eLoc-System mit einer Umgebungskarte sowie zurzeit mit einer auf Infrarot basierender Steuerung zum Auffinden seiner Ladestation (Abb. 5).

Die gesammelten Informationen werden, zusammen mit den Daten zu Kontaktaufnahme (Adresse, Straßenkarte, Wegbeschreibung) und evtl. der zu Hause gespeicherten medizinischen Information (Patientenakte) an die Kontaktstelle übermittelt. 
Abb. 5 Roboter mit Webcam am Unfallort
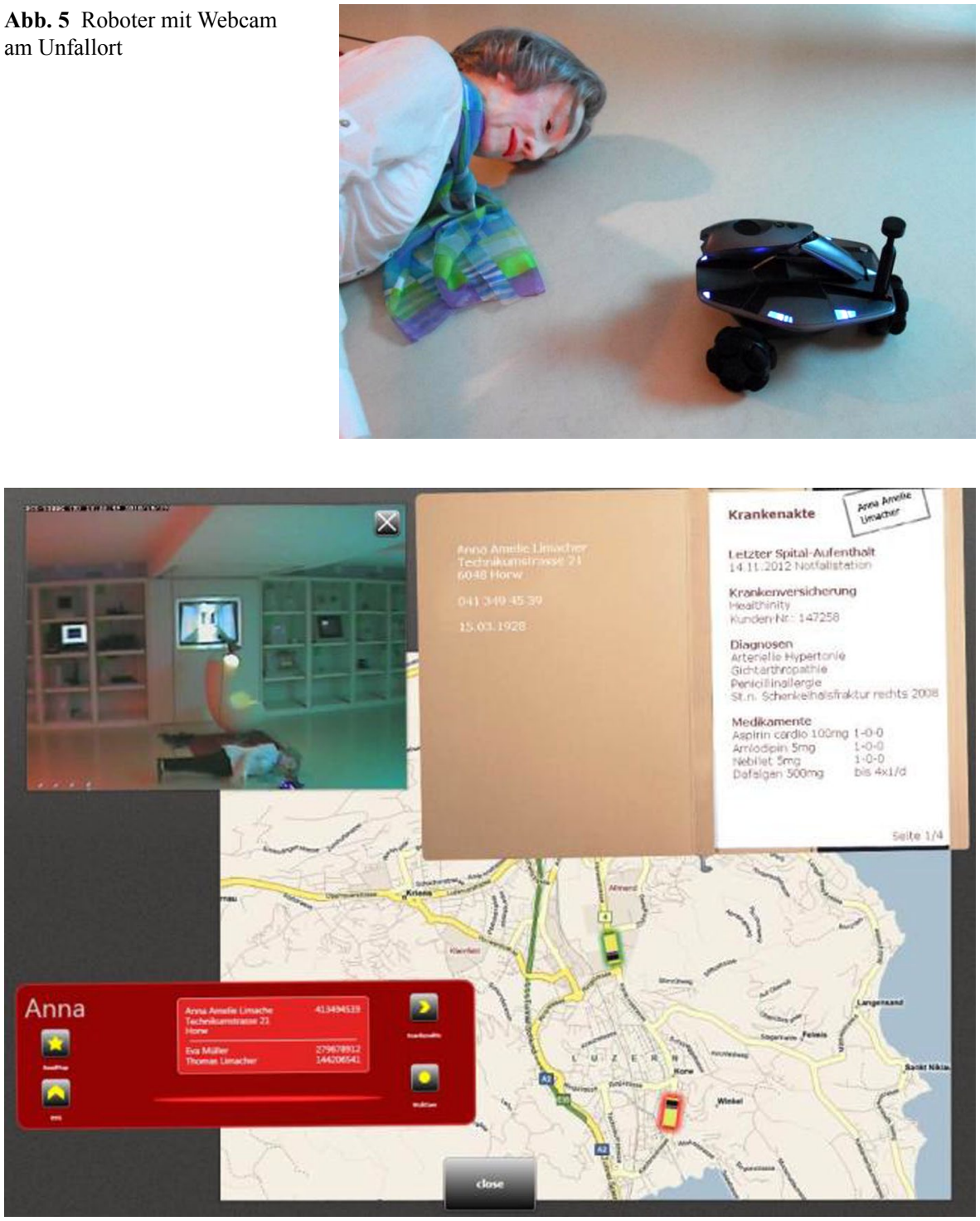

Abb. 6 iHomeTable Screeenshot bei der Einsatzplanung in der Notrufzentrale mit relevanten Informationen wie z. B. der Krankenakte

Das entwickelte Alarmsystem ist dabei so ausgelegt, dass die übermittelte Information den Anforderungen der Situation und der gewählten Kontaktstelle gerecht wird. Eine umfassende aber dennoch intuitive Oberfläche soll es den Operateuren erlauben, jederzeit den Überblick zu behalten, bei Bedarf in die Tiefe zu gehen und in Alarmsituationen gemeinsam schnell zu reagieren. Denkbar ist hier auch die Übermittlung aktueller Vitaldaten der verunfallten Person durch ein Tele Monitoring-System, dessen Anbindung sich noch in der Konzeptphase befindet (Abb. 6). 
Das entwickelte Konzept des iHomeRescue ermöglicht es leider nicht die Sturzprevention, kann aber die Folgen eines Sturzes durch ein zur Verfügung stehendes Rettungssystem mildern. Dies ist, ohne Einschränkung der Allgemeinheit, für ältere Menschen ein wichtiger Sicherheitsaspekt, der zu mehr Lebensqualität und einem selbstbestimmten Leben in der gewohnten Umgebung führen kann und dazu führt, dass ein Heimeintritt später erfolgen kann. Im iHomeLab der Hochschule Luzern konnte gezeigt werden, wie praxisnah ein solches System zum Einsatz kommen kann und welche technologischen Konzepte notwendig sind um ein multimediales Sicherheitskonzept zu realisieren.

\section{Literatur}

Andrushevich A, Kistler R, Bieri M, Klapproth A (2009) ZigBee/IEEE 802.15.4 Technologies in Ambient Assisted Living Applications; 3rd European ZigBee Developer's Conference (EuZDC), Munich, June 2009

Börsch-Supan A, Mariuzzo F (2005) Our Sample: 50+ in Europe [research report] Survey of Health, Ageing and Retirement in Europe, Vol. 2

Doblhammer G, Scholz RD, Maier H (2005) Month of birth and survival to age 105+: evidence from the age validation study of German semi-supercentenarians. Exp Geront 40(10):829-835. http://www. sciencedirect.com/science/article/pii/S0531556505001543

Entwicklung der Privathaushalte (2011) Statistisches Jahr-buch 2011, Statistisches Bundesamt, S. 63

Erdt S, Linner T et al (2011) Systematische Entwicklung eines komplexen Assistenzsystems zur Gesundheitsförderung am Beispiel des GEWOS-Bewegungssessels, 5. Deutscher AAL Kongress

IFA stellt Smart Homes in den Mittelpunkt. http://www.enbausa.de/lueftung-klima/aktuelles/artikel/ifastellt-smart-homes-in-den-mittelpunkt-3024.html. Zugegriffen: 17.01.2015

Knauth S, Jost Ch, Klapproth A (2009) Range sensor data fusion and position estimation for the iLoc indoor localisation system; ETFA 2009, WiP-1- Intelligent Robots and Systems, Palma de Mallorca, September 2009

Maier H, Gampe J, Vaupel JW, Jeune B (2010) Supercentenarians. Springer, Berlin

Mattern F (2007) Die Informatisierung des Alltags: Leben in smarten Umgebungen. Springer, Berlin

Naganawa H (1997) The work of the elderly and the silver human resources centers. JLB 36/6:5-7

Sayago S, Sloan D, Blat J (2011) Everyday use of computer-mediated communication tools and its evolution over time: an ethnographical study with older people interacting with computers 23:543-554

Schaible S, Kaul A, Lührmann M, Wiest B, Breuer P (2007), Wirtschaftsmotor Alter [Studie]. Bundesministerium für Familie, Senioren, Frauen und Jugend, Berlin. www.bmfsfj.de Geschäftsstelle: ,,Wirtschaftsfaktor Alter“ Jägerstrasse 67-69, Berlin. www.wirtschaftsfaktor-alter.de http://www.bmfsfj. de/RedaktionBMFSFJ/Broschuerenstelle/Pdf-Anlagen/Wirtschaftsmotor-Alter,property=pdf,bereich $=$,sprache $=$ de,rwb=true.pdf

Speziell im hohen Alter wird die Wohnung zum Lebensmittelpunkt, Francois Höpflinger. http://www. derbund.ch/bern/stadt/Speziell-im-hohen-Alter-wird-die-Wohnung-zum-Lebensmittelpunkt/story/ 13675491?track. Zugegriffen: 18.01.2015

Sundín E (2009) Life-cycle perspectives of product service systems. In: Sakao T, Lindahl M (Hrsg) Introduction to product/service-system design. Springer-Verlag, London

Voelcker-Rehage C, Godde B, Staudinger UM (2010) Physical and motor fitness are both related to cognition in old age. Eur J Neurosci 31:167-176

Wessig K (2012) Integrierte Versorgungskonzepte und Technologien in „Pflege 2020“, Fraunhofer Instituts für Arbeitsorganisation IAO Stuttgart 